\title{
Automated data collection using bar code
}

\author{
JOHN M. EILER, WALTER W. NELSON, CRAIG C. JENSEN, and STEVEN P. JOHNSON \\ Fircrest School, Seattle, Washington
}

\begin{abstract}
Using bar code technology to automate data collection provides a rapid and reliable alternative to paper-and-pencil tracking or keyboard entry into pocket or laptop computer. An array of bar code symbols is printed on a data menu, with a unique symbol corresponding to each possible observation. Bar code symbols are scanned using hand-held readers, which record the event, $\log$ the observation time, and store data for transfer to a personal computer. Advantages of the bar code monitoring system include: (1) ease of use by staff with minimal technical training, (2) reduced data entry errors and increased entry speed, (3) reliable portable operation, and (4) lowcost hardware. While the bar code system described here is used for behavioral monitoring in a residential treatment setting for the developmentally disabled, with minimal modification this system can be adapted for use in a wide variety of research and clinical applications.
\end{abstract}

Recording behavioral observations in research and institutional treatment settings has typically involved the use of paper-and-pencil forms, which must be routinely collected and tabulated prior to computer analysis. In addition to the administrative cost of maintaining treatment information by hand (which removes staff from other tasks), following a cumbersome monitoring routine can increase error in the data management system. Examples include failure to record events when they occur, inaccurate or incomplete recall of observations that occurred earlier in the day, deliberate entry of false data, transcription or calculation errors, and failure to provide thorough or timely data analysis. In recognizing these difficulties, treatment professionals are often forced to reduce their data collection requirements in an effort to obtain reliable data on a limited subset of available observations.

Directly keying observations into a computer system as events occur provides obvious advantages in reliability and avoids the expense and error involved in transcribing data from paper-and-pencil forms. Laptop computers offer the potential advantage of entering observations into the same personal computer program used for data analysis, but expense (ranging from $\$ 700$ to $\$ 3,000$ ) and portability (from 3 to $15 \mathrm{lb}$ ) limit their use, particularly in applications requiring continuous data entry from changing locations. Calculator-size pocket computers are better suited to portable event recording and can offer an affordable option (with costs as low as $\$ 120$ for some models). Pocket computer use typically requires keyboard entry of codes corresponding to various observations, tem-

\footnotetext{
The authors wish to thank Geoffrey $R$. Loftus for helpful comments on an earlier version of the manuscript. The support of Norm Davis, Superintendent of Fircrest School, made development of this system possible. We would also like to acknowledge helpful input from Lynne Daurelle, Gordon DeFalco, and Tore Lyderson regarding data systems design. Reprints can be obtained from John M. Eiler, Director, Training Resource Center, Fircrest School, Department of Social \& Health Services, 15230 15th Ave. N.E., Seattle, WA 98155.
}

porary RAM storage of these data, and transfer of data files to a personal computer for analysis. Developing and modifying the software necessary for these tasks requires custom programming, however, and may present a drawback for some users.

Current bar code technology can facilitate computer data collection by replacing keyboard entry of raw data. Although bar code use is becoming increasingly common in commercial inventory management applications (such as supermarket checkout stands), most researchers are not yet familiar with the potential benefits of bar code strategies for rapid coding and recording of behavioral observations. At its most basic, a bar code system consists of (1) printed symbols that represent data characters as a machine-readable pattern of parallel bars and spaces, and (2) a scanner that optically senses the symbol's pattern and recodes the signal as a sequence of text characters for computer input. The bar code data collection system described below demonstrates advantages over laptop and pocket computers in ease of use, accuracy, portability, and cost-effectiveness.

In contrast to commercial applications that affix a unique single-symbol bar code label to each item in inventory, behavioral observations are recorded from multiple bar code symbols printed on a one-page entry form, or data menu (see Figure 1). A unique bar code symbol is assigned to each possible observation, and the research staff scan the corresponding symbol on the data menu to record the event and automatically log the observation time. A hierarchical data input sequence is employed, for example, wherein the first scan indicates the staff responsible for the observation or treatment, the second scan identifies the client, and subsequent scans describe such information as observational category, treatment type, or treatment outcome. The graphic layout of the data menu guides the research staff through the entry hierarchy, without requiring memorization of the actual code characters for each observation. The ease of automated data entry reduces staff training time from several days to develop 
reliable keyboard skills to several minutes using bar code input.

Bar code data entry is also more accurate than keyboard input (Harmon \& Adams, 1984). Tests with experienced keypunch operators typing mixed alphanumeric characters yielded error rates ranging from $0.42 \%$ to $0.48 \%$ of total keystrokes ( 1 error in every 108 to 230 characters). Accuracy assurance is designed into the coding algorithms of bar code, with "excellent" print quality reducing er- ror to below 1 character in 70,000,000 and "good" print quality allowing 1 character substitution in $2,700,000$. While bar code virtually eliminates substitution errors, all bar code systems occasionally require repeated scans to enter a code. Bar code systems routinely achieve at least an $85 \%$ first read rate (the number of times a code is successfully read on the first pass divided by total scan attempts) and a $99 \%$ second read rate (the number of times a successful read occurs in the first two passes divided

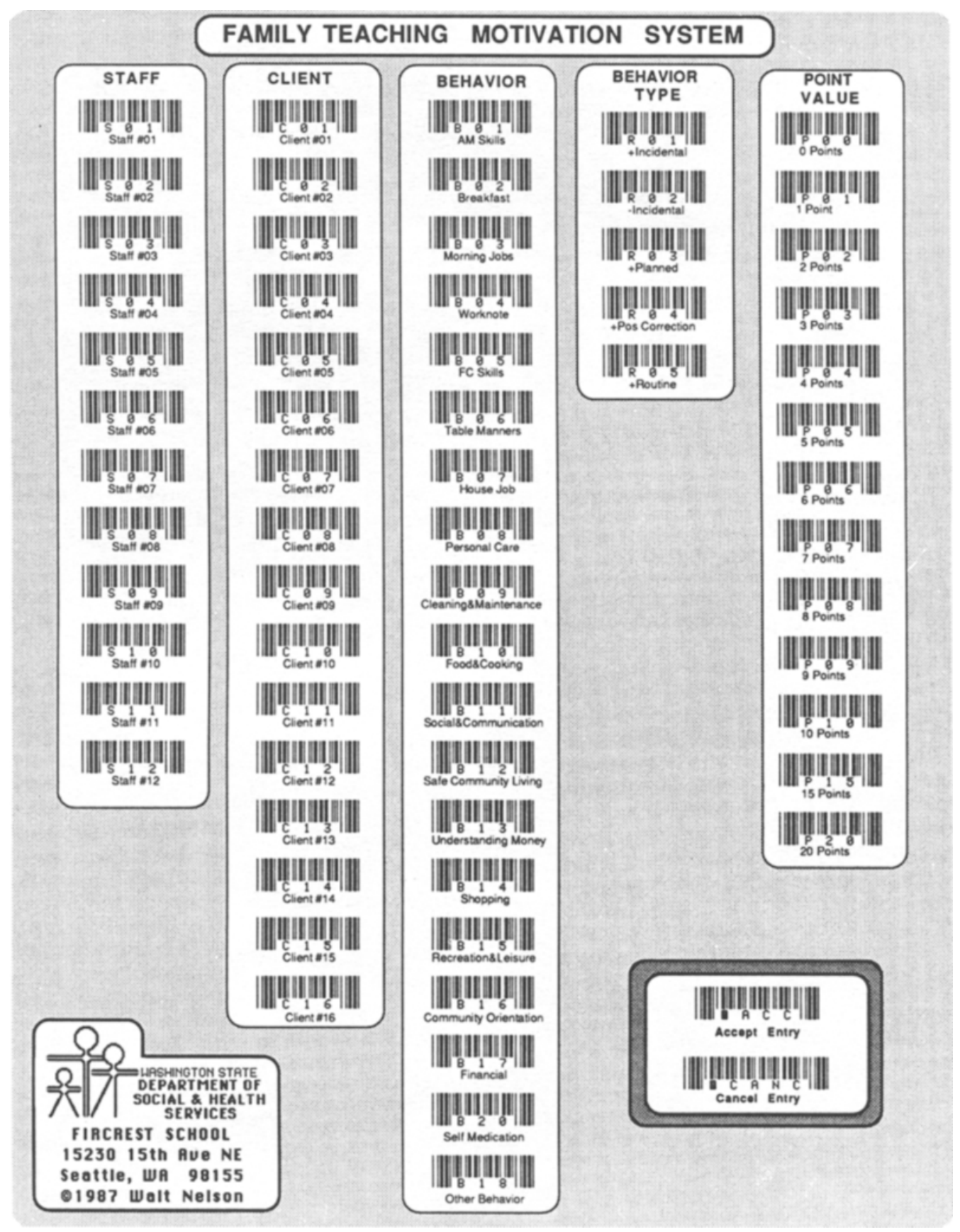

Figure 1. Bar code data entry menu used in "Family Teaching" token economy program. 
by total scan attempts). Improved overall data entry speed (4 characters/second for bar code vs. skilled keyboard entry rates of 2 characters/second) more than compensates for the occasional time loss to rescan a bar code symbol.

The hand-held scanners used in the current project are approximately the size of a credit card, offering a more portable solution to recording field observations than do laptop or pocket computers. Data menus can be mounted on walls and desktops or carried on clipboards and pocketsized binders. The scanners also can tolerate a more extreme range of environmental conditions than can computers, and have a longer service cycle between battery recharges.

With portable bar code systems available for under $\$ 400$, hardware prices are competitive with pocket computers and substantially less than laptop models. Since software is available for all Apple or IBM-compatible operating systems, existing personal computers and data management programs can be used. As detailed below, the ease of bar code system implementation can provide ongoing savings, particularly when used by staff having minimal technical experience.

\section{METHOD}

\section{Apparatus}

Scanner. The portable bar code scanner used in the current project was the TimeWand ${ }^{\mathrm{TM}}$ (by Videx, Inc.), an infrared light-sensing scanner measuring $3.37 \times 2.12 \times$ 0.31 in. and weighing $1.9 \mathrm{oz}$ (see Figure 2). The TimeWand uses a replaceable modular infrared optical sensor that offers excellent ambient light reflection and can operate in conditions ranging from complete darkness to direct sunlight. The scanner contains a low-power CMOS 8-bit microprocessor (NEC 80C49), in conjunction with rechargeable nickel-cadmium batteries that provide approximately 3 days of active use without recharging. An internal clock automatically records the date and time of each bar code scan, with a resolution of $15 \mathrm{sec}$. The TimeWand scanner also emits a variety of audible status signals (indicating correct scan, memory approaching capacity, memory full, data entered out of sequence, and entry of special "alert" codes), as well as a visible LED signal indicating correct scan.

TimeWand scanners are available in $2 \mathrm{~K}, 8 \mathrm{~K}$, and $16 \mathrm{~K}$ RAM sizes (at $\$ 198, \$ 228$, and $\$ 248$, respectively). The number of bar code scans available for storage at each configuration can be easily determined. A $16 \mathrm{~K}$ scanner has 15,872 bytes available for bar code storage, an $8 \mathrm{~K}$ has 7,680 , and the $2 \mathrm{~K}$ has 1,536 . Each bar code scan requires 1 byte per character plus 2 bytes overhead for recording the date and time . Additional programmable configurations (such as requirements for hierarchical data entry) can subtract between 256 and 512 bytes of available RAM storage. Therefore, in a typical application using six-character symbols without additional programmable options, a 2K TimeWand could gather 191 observations, an $8 \mathrm{~K}$ scanner could collect 959 , and the

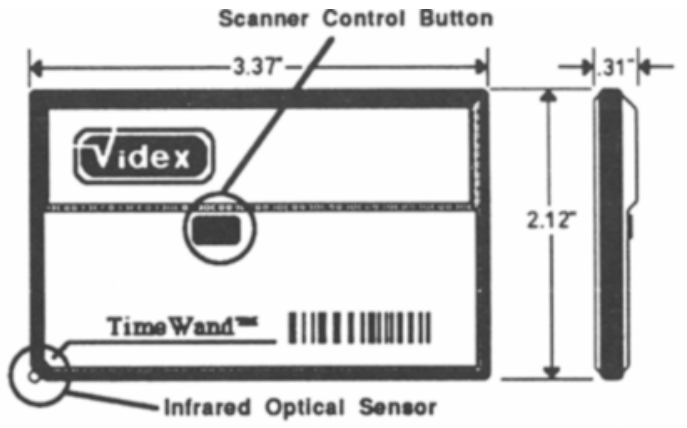

TimeWand ${ }^{\mathrm{TM}}$ Scanner

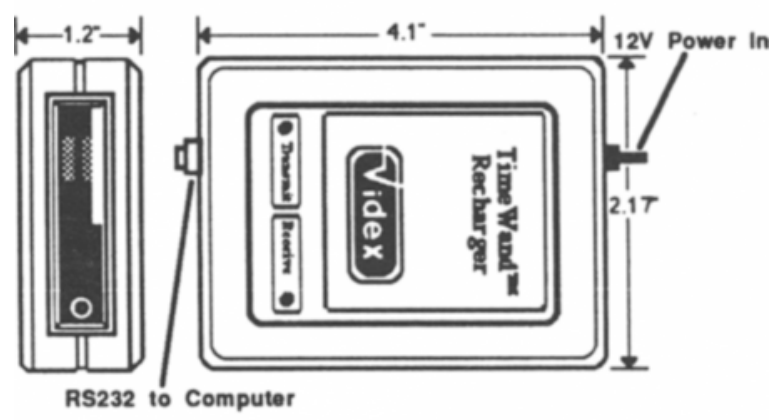

TimeWand ${ }^{\mathrm{M}}$ Recharger/Downloader

Figure 2. Videx TimeWand" hardware. The bar code scanner (upper) is operated by pressing the central button while moving the optical sensor across the bar code symbol. The scanner plugs into the recharging/download station (lower), which connects to the host computer or modem via RS232 cable.

16K TimeWand can hold 1,951 observations before downloading. A 16K TimeWand is capable of recording approximately one observation every $15 \mathrm{sec}$ for an 8-h period.

Downloader. The TimeWand system uses a recharging/download station $(\$ 149)$ that serves three functions. First, the unit transfers data from the scanner to the host computer via a serial RS232 port at 1,200 baud. A "transmit" status light indicates that downloading is in progress, and a checksum is used to verify that data are correctly transmitted. Both closed loop optical and contact communications techniques are employed for data communication. Second, the station allows the host computer to initialize the TimeWand and configure it to include an ID number, data file name, data entry options (such as hierarchical sequence restrictions), and the bar code symbology employed. The scanner's internal clock is reset by the host computer clock after each download. A "receive" status light indicates configuration activity. Third, the downloader also serves as a recharging unit, using a 12$\mathrm{V}$ adapter/power supply that requires 10 to $14 \mathrm{~h}$ to fully charge a completely discharged battery.

TimeWand recharging/download stations measure 4.1 $\times 3.12 \times 1.2$ in. (see Figure 2), or approximately the size of a cigarette pack. Multiple stations can be daisy- 
chained together to a single serial port, or more economical modular units are available to maintain up to 25 scanners from a single computer installation. The recharging/download stations can also be connected to standard RS232 modems for remote downloading capability via telephone lines.

Host computer. Our current project uses several Apple Macintosh Plus computers (1 Mb RAM) with either single or double $800 \mathrm{~K}$ disk drives, although Videx currently provides software support for the TimeWand system on IBM PC-compatible, Apple II, and TRS models 100 and 200 laptop computers as well. The downloading and configuring applications exist as a "desk accessory" utility in the Macintosh operating system. This utility creates and stores data with identifying header information, bar code characters and time stamps in standard ASCII text files that can be readily imported into most database management programs for subsequent data analysis and reports. The TimeWand Manager ${ }^{\mathrm{TM}}$ database program is available from Videx.

Printing. Over 60 symbologies exist for encoding bar patterns into data for computer input, with specific advantages and drawbacks to each (Allais, 1985; Harmon \& Adams, 1984). Our current project uses Code 39 because of its flexibility in representing both text and numeric character sequences of varying lengths, acceptable printing with conventional hardware, and robust error-checking structure that yields high first-read rates and extremely low character substitution rates. Although firmware support for Code 39 is provided in the TimeWand, the scanner can be programmed to read most popular bar code symbologies (i.e., UPC, interleaved 2 of 5 , EAN, Codabar, Code 128, etc.). Every bar code contains start/stop punctuation that defines the limits of the code, with data contained as ASCII character information between these marks. The TimeWand can scan codes from 0 to 23 characters in length, with an optimal length of up to 6 characters.

For flexibility in designing and modifying data collection sheets, Videx provides a bar code font for the Apple Macintosh computer that provides excellent results when codes are created with object-oriented graphics software, such as MacDraw (Claris Corp.). Although readable data sheets can be produced on a good quality dot matrix printer (such as the Apple Imagewriter), our current project uses laser output (from an Apple Laserwriter printer) that provides vastly improved first-read rates and enables precise reduction of the bar codes for various data menu specifications.

Bar codes should be covered with a thin laminate to protect the pattern from wear with repeated scanning. After experimenting with several laminating procedures, we use clear adhesive sheets to provide inexpensive protection of the bar code patterns without interfering with scanning accuracy.

\section{Procedure}

Fircrest School currently has 12 installation sites, providing bar code data entry for various systemic be- havioral treatment programs. Although the treatment modalities, training environments, staff characteristics, observation types, and report formats vary considerably across these sites, the following common elements were addressed in developing a bar code data management system for each application: (1) designing efficient data collection menus, (2) establishing procedures for transferring treatment information to computers and maintaining bar code system operations, and (3) devising automated data analysis and report routines to convey summary information to treatment professionals and to link results to existing databases.

Data menu. As described above, observations are recorded by scanning multiple bar code symbols printed on a comprehensive data entry form. In most of our installation sites, design of the data menu parallels the layout of existing checklist forms. It is not necessary, of course, to have separate forms for each client or multiple copies available with blank spaces for daily paper-andpencil data entry; a single input sheet will usually suffice to record a range of observations for multiple clients over extended periods of time. In most instances, the data menus can be quite concise, since the scanner automatically records the time and date of data entry, and staff identification can occur at the beginning of a shift with entry from a separate menu or from an employee's ID card or badge.

The characters that define each bar code symbol comprise an ASCII text string that represents coded information in a database. Each bar code symbol must be assigned a unique character string. If hierarchical scanning is desired (to prohibit scanning a symbol out of sequence), a unique first character must be assigned to identify each category of variable. For example, a teacher or client ID symbol may begin with the characters $\mathrm{T}$ or $\mathrm{C}$, followed by two digits that can represent up to 100 different instances of these categories. Although Code 39 symbols display the actual code in text characters beneath the bar pattern, each symbol on the data menu must also have a text label that more clearly defines the observation for the routine user (see Figure 1).

In treatment settings where it is critical to maintain the confidentiality of client data, bar code recording may have additional advantages over paper-and-pencil records. For example, placing client treatment forms on clipboards for easy access may violate regulations requiring locked storage of confidential medical records. It is acceptable, however, to allow open access to a bar code data entry form that contains no visible record of confidential client information. This permits the staff to record data without the time expense of unlocking a medical records cabinet, locating the correct client chart and treatment section, and entering data on the appropriate input form.

While certain records obviously need to be maintained in narrative form and are not amenable to bar code input, records of this type are not typically intended for computer storage and manipulation. Most data collection forms that require brief responses in predictable categories transfer readily to bar code entry. In fact, bar code observations can offer advantages by forcing user responses 
into predetermined, uniquely coded categories, thus eliminating idiosyncratic narrative responses. Although this facilitates data reduction and analysis, it becomes critical to anticipate all bar code symbols and combinations necessary to describe any potential information and to train staff in reliable classification of observations.

System maintenance. Data entry menus are produced at a central laser printer site, with initial layout and subsequent modifications performed by a technical consultant to the professionals responsible for designing individual treatment programs. Modifications are not usually needed more than once a month, but updating the database also requires altering the data entry menu.

The various Macintosh host computer sites on the Fircrest School campus are in administrative offices, separate from the direct client treatment areas. In most cases, the bar code scanners are delivered at the beginning and end of each work day by designated staff trained in the downloading procedure. The daily data files from each treatment site are downloaded in a simple 5-min procedure and, in most cases, automatically appended to a weekly raw data file. Staff with no prior computer experience are successfully trained in downloading, file storage, and recharging procedures in less than $2 \mathrm{~h}$.

Automated data management. While the initial benefits of implementing a bar code system are apparent in increased speed and accuracy at the point of data entry, appreciable gains are also realized in expediting subsequent information management. This secondary benefit accrues through reducing the efforts of staff responsible for transcribing, tabulating, summarizing, analyzing, and reporting the data. Raw data files are downloaded into the database program using a single "macro-command" that performs a series of sorts and summary procedures to produce standard reports. In addition to monitoring client progress, analysis of staff performance is also provided (see Figure 3). These reports are generated by various Macintosh database applications and are tailored to the monitoring requirements of the professionals responsible for the treatment site. The automated data entry and report generation procedures are performed by administrators or treatment staff with no prior computer experience and after less than $2 \mathrm{~h}$ of technical training.

\section{RESULTS AND EVALUATION}

Direct measures of time savings were available in pilot installations where a bar code system replaced an existing paper-and-pencil data collection strategy. Previous data management systems required an average of $36 \mathrm{~h} / \mathrm{month}$ to tabulate, summarize, analyze, and enter the treatment information required for the monthly client progress report. Automated bar code systems reduced this process to an average of $3 \mathrm{~h} / \mathrm{month}$. At a prorated labor rate of $\$ 8.50 / \mathrm{h}$ for the job classifications performing these tasks, average monthly savings of $\$ 280.50$ were realized, with projected annual reductions of $\$ 3,366$ for each installation site.

Equipment costs based on retail prices of $\$ 248$ for a $16 \mathrm{~K}$ scanner and $\$ 149$ for a recharging/download station

\section{Family Teaching Data Summary From 1/10/88 To 1/10/88}

\begin{tabular}{|c|c|c|c|c|c|c|c|}
\hline Staff & $\begin{array}{c}\text { \# of } \\
\text { Interactions }\end{array}$ & $\begin{array}{c}\% \text { of } \\
\text { Interactions }\end{array}$ & $\stackrel{+}{\stackrel{+}{\text { Incidentals }}}$ & Incidentals & $\stackrel{+}{+}$ & $\stackrel{+}{+}$ & $\begin{array}{c}+ \\
\text { Planned }\end{array}$ \\
\hline Staff \#01 & 10 & $28.6 \%$ & 1 & 0 & 0 & 8 & 1 \\
\hline Staft \#02 & 17 & $48.6 \%$ & 1 & 2 & 1 & 12 & 1 \\
\hline $\begin{array}{l}\text { Staff } \# 03 \\
\text { Overall: }\end{array}$ & $\frac{8}{35}$ & $22.9 \%$ & $\frac{2}{4}$ & $\begin{array}{l}2 \\
2\end{array}$ & $\frac{0}{1}$ & $\frac{6}{26}$ & $\frac{0}{2}$ \\
\hline
\end{tabular}

Percent of Negative Interactions Resulting in Positive Correction $=\mathbf{5 0 . 0 \%}$

Amount of Teaching

Total Number of Interactions

Avg. Daily Interactions per Client 11.7

$\begin{array}{rccc}\text { RIP Distribution: } & \text { Routine } & \text { Incidental } & \text { Planned } \\ \text { \# of Interactions } & 26 & 7 & 2 \\ \% \text { of Interactions } & 74.3 \% & 20.0 \% & 5.7 \%\end{array}$

\begin{tabular}{|c|c|c|c|c|c|c|c|}
\hline Client & $\begin{array}{c}\text { Positive } \\
\text { Interactions }\end{array}$ & $\begin{array}{l}\text { Negative } \\
\text { Interactions }\end{array}$ & Ratio & $\begin{array}{c}\% \\
\text { Pos }\end{array}$ & $\begin{array}{r}\% \\
\text { Nea }\end{array}$ & $\begin{array}{l}\text { Point } \\
\text { Balance }\end{array}$ & $\begin{array}{l}\% \text { of Total } \\
\text { Balance }\end{array}$ \\
\hline Client $\# 01$ & 16 & 2 & 8.0 & $48 \%$ & $100 \%$ & 36 & $36.4 \%$ \\
\hline Client \#02 & 8 & 0 & NA & $24 \%$ & $0 \%$ & 30 & $30.3 \%$ \\
\hline $\begin{array}{l}\text { Client \#03 } \\
\text { Overall: }\end{array}$ & $\frac{9}{33}$ & $\frac{0}{2}$ & $\frac{N A}{16.5}$ & $\frac{27 \%}{94 \%}$ & $\frac{0 \%}{6 \%}$ & $\frac{33}{99}$ & $33.3 \%$ \\
\hline \multicolumn{8}{|c|}{ Mean Ratio $=\mathbf{8} .0$} \\
\hline
\end{tabular}

Figure 3. Sample daily report from "Family Teaching" token cnonomy program. Note that both staff and client behaviors are described and that the daily client point balance is reported for token exchange. 
equal $\$ 796$ for the average two-scanner installation. Since existing host computers were utilized, the hardware costs for each site were recovered within 3 months of operation. Hardware reliability has been excellent, with replacement of one missing scanner and less than $\$ 100$ in equipment repairs during our initial implementation of 12 sites.

The above cost/benefit figures are conservative estimates. They do not account for time spent in data entry, which contrasts bar code scans to recording observations on paper-and-pencil forms located in individual client charts stored in locked medical record cabinets. Staff training costs in bar code entry are equivalent to or somewhat less than training new staff in a variety of existing forms and should be recovered in less than a year in a facility with relatively frequent staff turnover or reassignment.

While qualitative measures of improved accuracy, reliability, and effectiveness of program monitoring are more difficult to assess, clear benefits to clients, direct care staff, professionals, and management personnel have been realized in the following areas: (1) staff spend less time in data management chores, releasing them for more productive and rewarding time in direct client interaction; (2) client progress reports are issued in a more timely fashion, expediting the loop of providing, monitoring, and modifying treatment programs; (3) the accuracy and level of measurement in data collection have been substantially improved, providing more meaningful treatment reports and resulting in an improved information base for professional decisions; (4) objective measures are available for behavioral staff-management systems (e.g., Frederiksen, 1982) that provide staff with frequent and timely feedback and reinforcement based on their performance; (5) improvements in morale have been noted in staff, who express satisfaction in using an innovative treatment tool, developing new technical skills, pursuing professional opportunities for research, and participating in a model management project that they can expand to other applications.

\section{DISCUSSION}

Along with increasing the number of installation sites, other modifications to the current bar code system are being considered. A download/recharging station can be cabled to a modem, allowing raw data files from scanners at remote sites to be transferred to a host computer over existing telephone lines. This strategy has particular appeal if selected clients can learn to self-monitor various aspects of their own treatment using a bar code scanner and relay these data to the institution from an off-campus residence. Further automation is also possible by using the database management program to produce data menus. In this way, modification to codes in the database could result in updated bar code symbols for data entry, as opposed to requiring separate changes to data menus using a graphics program.

Many applications requiring rapid, reliable monitoring and documentation of observable events, staff performance, or treatment delivery may benefit from the increased efficiency that the bar code system provides. The 15 -sec resolution of the scanner's internal clock may present a limitation in some research settings, however. This clock speed was a design compromise to permit logging times over the entire recharge cycle of the scanner's battery; portable scanners with faster clock speeds may become available in the future. Hand-held bar code scanners do have an inherent 2- to 3-sec interval necessary to align the read head and scan a symbol, but since entry results in input of several characters, this still represents faster input than via keyboard.

It is not possible in this brief overview to summarize the various applications of bar code technology to research and clinical settings. The use of computer automation to improve the speed, accuracy, and reliability of data management offers obvious benefits for most behavioral scientists, and wide-ranging strategies have long been available for this purpose. Bar code systems, however, can deliver additional advantages in ease of use, accurate data entry, portability, and cost-effectiveness, and should spread rapidly as this technology becomes more familiar.

\section{REFERENCES}

AlLAIS, D. C. (1985). Bar code symbology: Some observations on theory and practice. Lynnwood, WA: INTERMEC.

FREDERIKSEN, L. W. (Ed.) (1982). Handbook of organizational behavior management. New York: Wiley.

Harmon, C. K., \& Adams, R. (1984). Reading between the lines: An introduction to bar code technology. Peterborough, NH: Helmers Publishing.

(Manuscript received July 15, 1988; revision accepted for publication November 1, 1988.) 